\title{
Diagnostic Accuracy of PIVKA-II, Alpha-Fetoprotein and a Combination of both in Diagnosis of Hepatocellular Carcinoma in Patients Affected by Chronic HCV Infection
}

\author{
IVAN GENTILE ${ }^{1}$, ANTONIO RICCARDO BUONOMO ${ }^{1}$, RICCARDO SCOTTO $^{1}$, EMANUELA ZAPPULO ${ }^{1}$, \\ CANIO CARRIERO ${ }^{1}$, MAURO PICCIRILLO ${ }^{2}$, FRANCESCO IZZO ${ }^{2}$, MARIANNA RIZZO ${ }^{3}$, \\ DIONIGIO CERASUOLO ${ }^{3}$, GUGLIELMO BORGIA ${ }^{1}$ and ERNESTA CAVALCANTI ${ }^{3}$ \\ ${ }^{1}$ Section of Infectious Diseases, Department of Clinical Medicine and Surgery, \\ University of Naples "Federico II", Naples, Italy; \\ ${ }^{2}$ Division of Abdominal Surgical Oncology, Hepatobiliary Unit, \\ "Fondazione G. Pascale" National Cancer Institute, IRCCS, Naples, Italy; \\ ${ }^{3}$ Division of Laboratory Medicine, "Fondazione G. Pascale" National Cancer Institute, IRCCS, Naples, Italy
}

\begin{abstract}
Background/Aim: Patients affected by liver cirrhosis are at high risk for developing hepatocellular carcinoma (HCC). The aim of this study was to evaluate the feasibility of PIVKA-II (protein induced by vitamin K absence or antagonist-II) alone or in combination with $\alpha-1$ fetoprotein $(A F P)$, as a screening marker for development of HCC. Materials and Methods: A case-control study was conducted in 2 hospital wards in Naples. All anti-HCV-positive patients affected by HCC were considered as cases, while consecutive anti-HCV-positive patients without $H C C$ were considered as controls. Results: Overall, 160 patients were enrolled, 56 cases and 104 controls. At the set cut-off of $36 \mathrm{mAU} / \mathrm{ml}$, PIVKA-II was more sensitive (78.6\% vs. 60\%), but less specific than AFP at the set cut-off of $12 \mathrm{ng} / \mathrm{ml}(66.3 \% \mathrm{vs}$. $77.2 \%)$. The negative predictive value of PIVKA in combination with AFP was 93.2\%. Conclusion: PIVKA II, when combined with AFP, may be considered as a screening test for HCC due to its high negative predictive value.
\end{abstract}

Hepatitis C virus (HCV) infection affects more than 185 million people worldwide and is estimated to cause about 350,000 deaths every year (1).

This article is freely accessible online.

Correspondence to: Antonio Riccardo Buonomo, Department of Clinical Medicine and Surgery, Section of Infectious Diseases, "Federico II" University of Naples, Via S. Pansini 5, 80131 Naples, Italy. Tel: +39 081/7463740, e-mail: antonioriccardobuonomo@ gmail.com

Key Words: Cirrhosis, HCV, PIVKA-II, AFP, HCC.
HCV can cause both acute and chronic hepatitis. In 55$85 \%$ of cases, acute HCV infection becomes chronic. Chronic hepatitis $\mathrm{C}$ infection progresses towards cirrhosis in $15-30 \%$ of cases in about 20 years and about $2-4 \%$ of patients with cirrhosis develop hepatocellular carcinoma (HCC) each year $(1,2)$. HCC is the fifth most common cancer in males and the seventh in females, causing up to 700,000 deaths each year (3). Because of the high risk of developing HCC, per authoritative guidelines, patients with cirrhosis should undergo periodic screening with abdominal ultrasound to detect HCC at an early stage.

Both the American Association for the Study of Liver Diseases (AASLD), and the European Association for the Study of the Liver/European Organization for Research and Treatment of Cancer (EASL-EORTC) produced guidelines on the diagnosis, management and surveillance protocols of HCC $(2,4)$. Both guidelines, with due differences, agree on screening of at-risk patients at least every six months with abdominal ultrasonography. There is no consensus on the use of serum biomarkers.

The most commonly utilized biomarker in clinical practice for HCC is $\alpha-1$ fetoprotein (AFP). AFP may be used as a diagnostic and prognostic marker. Serum AFP levels also correlate with tumor size, tumor doubling time and posttreatment relapse $(2,5-8)$. However, it is not recommended for routine screening because it lacks specificity. AFP may be elevated in other malignancies (e.g. gastric, embryonic cancer, intrahepatic cholangiocarcinoma), as well as in many benign conditions, both hepatic (e.g. cirrhosis, necrosis, acute hepatitis), and extrahepatic (e.g. pneumonia). Finally, AFP lacks sensitivity; serum AFP levels are high in only 40-60\% of HCC cases and only 10-20\% of early HCC (5). For these reasons, more sensitive and specific HCC markers are required. 
PIVKA-II (protein induced by vitamin $\mathrm{K}$ absence or antagonist II), also known as des-gamma-carboxyprothrombin (DCP), is an immature form of prothrombin without any coagulative function. It is synthesized in the presence of acquired defects of precursor carboxylation found in patients with HCC $(5,10)$. PIVKA-II may have a role in HCC progression by acting as an autologous growth factor (11-13). Moreover, high serum levels of PIVKA-II are associated with HCC size, microvascular invasion, metastatic dissemination and $\mathrm{HCC}$ relapse after liver transplantation and/or HCC nodule ablation $(8,14-17)$. PIVKA-II is a specific marker for $\mathrm{HCC}$, it is poorly related to AFP and exhibits higher sensitivity and specificity than AFP in diagnosing HCC. PIVKA II is not elevated in benign hepatic diseases (e.g. cirrhosis, necrosis) $(10,18)$, while it is elevated in HCC at early stage as well as in HCC nodules of all sizes $(8,18,19)$.

For these reasons, some authors, and especially Japanese guidelines on HCC management proposed the use of PIVKA-II for routine follow-up in patients with high risk for developing HCC $(5,20)$, as well as a prognostic biomarker before surgery to predict microvascular invasion, relapse and overall poor prognosis (14-17). In detail, Japanese guidelines have suggested to consider as a diagnostic marker of HCC high elevation of tumor markers, namely AFP $\geq 200 \mathrm{ng} / \mathrm{ml}$ and PIVKA-II $\geq 40 \mathrm{mAU} / \mathrm{ml}$.

However, data on its performance as a diagnostic marker are still incomplete. The aims of the study were to evaluate: i) the diagnostic accuracy of PIVKA-II in the diagnosis of $\mathrm{HCC}$ in HCV-related liver cirrhosis; ii) the comparative diagnostic accuracy of PIVKA-II and AFP in the diagnosis of HCC; iii) the diagnostic accuracy of a novel marker derived from the association of AFP and PIVKA-II.

\section{Materials and Methods}

We conducted a case-control study performed in 2 hospital wards, the Infectious Disease Unit of the "A.O.U. Federico II" of Naples and the Hepatobiliary Surgery Unit of the "Fondazione G. Pascale" National Cancer Institute.

Study population. All patients aged 18 and above admitted to the participating hospital wards that met the following inclusion criteria were enrolled in the study;

- Cases: proven HCV infection (positive anti-HCV and detectable serum HCV-RNA) plus radiological, histological or cytological evidence of hepatocellular carcinoma as assessed in AASLD hepatocellular carcinoma guidelines (2). Exclusion criteria were consent refusal, previous pharmacological or local HCC therapy and anticoagulant therapy.

- Controls: infection with HCV (positive anti-HCV and detectable serum HCV-RNA), negative history for HCC and absence of nodular hepatic lesions under abdominal ultrasonography.

All patients underwent history-taking, physical examination, routine laboratory examinations, serological testing for $\mathrm{HCV}$ and
HBV infections and abdominal imaging either by abdominal ultrasonography, contrast-enhanced CT- scanning or contrastenhanced MRI according to the clinical judgment. Cases were also categorized according to CLIP (The Cancer of the Liver Italian Program) staging score. Blood samples for AFP and PIVKA-II assays were also collected from all the enrolled patients, regardless if they belonged in the case or in the control group. PIVKA-II assay was performed using Lumipulse ${ }^{\circledR}$ G1200 (Fujirebio Inc., Malvern, PA, USA), an enzyme-linked immunoassay based on chemiluminescence principles (CLEIA - chemiluminescent enzyme immunoassay). Tests were performed at the Laboratory Medicine unit of the "Fondazione G. Pascale" National Cancer Institute.

Ethical aspects. The study was conducted in accordance with Good Clinical Practices and was regulated by the latest revision of the Helsinki Declaration. Data handling was in accordance with the Italian law on privacy. All enrolled patients were requested to sign a written consent after being informed about the study aims. The patients were managed per best routine clinical practice. The study was first approved by the Ethical Committee of the coordinating clinical unit Ethical Committee of University of Naples "Federico II".

Statistical analysis. All patients enrolled were included in the analyses. Continuous variables were reported as mean \pm SD (standard deviation) or as median and interquartile range (IQR) when appropriate. Between-group comparisons were made using T-student test and Mann-Whitney $U$-test when appropriate. Categorical variables were reported as percentages. Among-group comparisons were made using the chi-squared test or the Fisher's exact test when appropriate. The cut-off for statistical significance was set at $5 \%$ in two-tailed test. The diagnostic accuracy was assessed using ROC curve. Correlation of selected variables was evaluated using Pearson's correlation coefficient.

We categorized PIVKA-II and AFP serum levels to obtain a sensitivity rate of $80 \%$ and we also performed a composite score between PIVKA-II (categorized to $36 \mathrm{mAU} / \mathrm{ml}$ ) and AFP (categorized to $12 \mathrm{ng} / \mathrm{mL}$ ) and we tested diagnostic accuracy of this score in the diagnosis of HCC.

Statistical analysis was performed using software package SPSS 18.0: PASW Statistic (SPSS, Inc, Chicago, IL, USA).

\section{Results}

Baseline characteristics of the 160 enrolled patients are shown in Table I. Significant differences in socio-demographic variables between cases and controls were observed for gender $(p=0.004)$ and age $(p=0.002)$. No differences were observed in clinical variables between the two groups. For laboratory variables, significant differences were observed in glucose levels $(p<0.001)$, albumin levels $(p=0.003)$, platelet count $(p=0.038)$ and serum HCV-RNA $(p=0.014))$. Both serum AFP (17.3 vs. $5.1 \mathrm{ng} / \mathrm{ml}, \quad p<0.001)$ and serum PIVKA-II concentrations $(146.5 v s .27 .5, p<0.001)$ were higher in the case group (Table I). Serum levels of AFP and PIVKA-II were not correlated ( $\varrho=0.146)$ (Figure 1).

The performance of both PIVKA-II and AFP in the diagnosis of HCC were plotted on a ROC curve (Figure 2) showing similar areas under curve for both markers; 
Table I. Characteristics of patients $(n=160)$.

\begin{tabular}{lccc}
\hline & Cases $(\mathrm{n}=56)$ & Controls $(\mathrm{n}=104)$ & $p$-Value \\
\hline Males & $41(73.2 \%)$ & $59(56.7 \%)$ & 0.004 \\
Age (years) & $70(65-77)$ & $66(57-72)$ & 0.002 \\
Child-Pugh class & $\mathrm{A}=40(71.4 \%)$ & $\mathrm{A}=48(64.9 \%)$ & 0.604 \\
& $\mathrm{~B}=14(25.0 \%)$ & $\mathrm{B}=20(27.0 \%)$ & $\mathrm{C}=6(8.2 \%)$ \\
MELD score & $\mathrm{C}=2(3.6 \%)$ & $9.70(7.50-12.90)$ & 0.433 \\
Platelet count (elements/mmc), & $9(7.90-11.80)$ & $131,000(88,000-189,750)$ & 0.038 \\
Haemoglobin (g/dl) & $108,500(74,500-149,000)$ & $13.80(11.40-15.00)$ & 0.097 \\
INR & $12.90(12-14.20)$ & $1.10(103-1.22)$ & 0.208 \\
Ferritin (ng/ml), & $1.14(1.06-1.26)$ & $201(93-403)$ & 0.926 \\
Glucose (mg/dl) & $255(34-414)$ & $94(80-116)$ & $<0.001$ \\
Albumin (g/dl), & $110(100-44)$ & $3.90(3.50-4.40)$ & 0.003 \\
Total Bilirubin(mg/dl) & $3.70(3.20-3.90)$ & $0.90(0.60-1.50)$ & 0.929 \\
AST (U/1) & $0.90(0.60-1.30)$ & $57(37-92)$ & 0.261 \\
ALT (U/l) & $59(31-118)$ & $54(34-87)$ & 0.750 \\
Log HCV-RNA (UI/ml) & $47(30-107)$ & $5.94(5.31-6.39)$ & $<.014$ \\
AFP (ng/mL) & $5.20(4.55-5.67)$ & $5.10(2.90-11.40)$ & $<0.001$ \\
PIVKA-II (mAU/ml) & $17.30(6.50-159)$ & $27.50(21.20-48)$ & $<0.001$ \\
\hline
\end{tabular}

Data are expressed as median (interquartile range) for quantitative variables or number of cases (percentage) for qualitative ones. $*$ Child-Pugh and MELD are calculated on patients with clinical cirrhosis.

specifically, areas under curve (AUC) for PIVKA-II (cutoff:36 mAU/ml) and AFP (cut-off:12 $\mathrm{ng} / \mathrm{ml}$ ) were 0.788 $(95 \% \mathrm{CI}=0.707-0.868)$ and $0.756 \quad(95 \% \mathrm{CI}=0.676-0.836)$ respectively. At the set cut-off value of $36 \mathrm{mAU} / \mathrm{ml}$, PIVKAII was more sensitive $(78.6 \% \mathrm{vs} .60 \%)$ but less specific than AFP at the set cut-off value of $12 \mathrm{ng} / \mathrm{ml}(66.3 \% \mathrm{vs} .77 .2 \%)$. The negative predictive value of PIVKA in combination with AFP was $93.2 \%$ and it was higher when compared both to AFP (75.9\%) and PIVKA alone (85.2\%) (Table II).

All cases of HCC $(n=56)$ had liver cirrhosis. HCC was graded using CLIP staging score. Most cases (39/56 patients, $70 \%$ ) had a CLIP score of 0-1. Patients with CLIP score of 2 or $3-4$ were $18 \%(10 / 56)$ and $12 \%(7 / 56)$, respectively. Subgroup analysis of cases showed that patients with CLIP score 3-4 (vs. 0-2) had significantly higher serum levels of PIVKA-II (159 vs. $3.458 \mathrm{mAU} / \mathrm{ml}, p=0.02$ ) (Figure 3).

Similarly, patients with CLIP score 3-4 (vs. 0-2) had significantly higher serum levels of AFP (244 vs. 14.6 $\mathrm{ng} / \mathrm{mL}, p=0.023$ ) (Figure 4).

\section{Discussion}

HCC is the sixth most common cancer in the world and the third most common cause of cancer-related death. The prognosis of HCC worsens as CLIP score increases (21). In our study, patients with HCC were older and more often males than patients without HCC. Male predominance may be explained by greater exposure to toxins, $\mathrm{HCV}$ prevalence and hepatocyte androgenic stimulation (22). We also

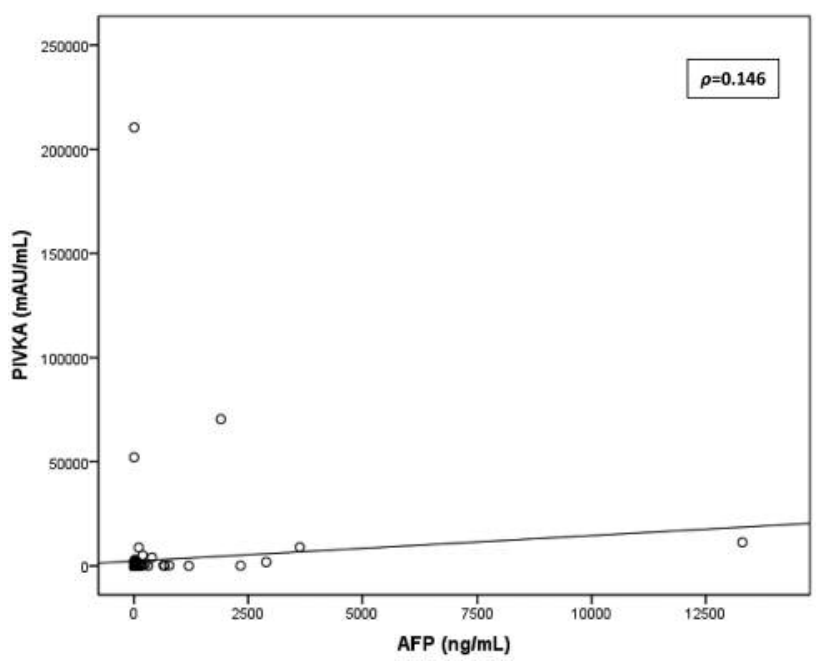

Figure 1. Linear correlation between AFP and PIVKA-II. PIVKA: prothrombin induce $\backslash$ d by vitamin $K$ absence-II; AFP: $\alpha$-fetoprotein; HCC: hepatocellular carcinoma.

observed higher blood glucose levels and lower levels of albumin and platelets; these results are in accordance with the role of metabolic syndrome and insulin-resistance as risk factors for $\operatorname{HCC}(6,23)$.

Patients with cirrhosis, especially if due to $\mathrm{HBV}$ or $\mathrm{HCV}$, are at high risk for developing $\mathrm{HCC}$ and, therefore, undergo active surveillance with six-monthly upper abdominal 




Figure 2. Receiver operating characteristics (ROC) curve comparing specificity and sensibility of PIVKA-II and AFP in detecting hepatocellular carcinoma in patients affected by $\mathrm{HCV}$ chronic liver disease. PIVKA: prothrombin induced by vitamin $K$ absence-II; AFP: $\alpha$-Fetoprotein; HCC: hepatocellular carcinoma.

Table II. Sensitivity, specificity, positive predictive value (PPV) and negative predictive value (NPV) of AFP, PIVKA-II and the combination of both in detecting $\mathrm{HCC}$.

\begin{tabular}{lccc}
\hline & $\begin{array}{c}\text { AFP> } \\
12 \mathrm{ng} / \mathrm{ml}\end{array}$ & $\begin{array}{c}\text { PIVKAII> } \\
36 \mathrm{mAU} / \mathrm{ml}\end{array}$ & $\begin{array}{c}\text { AFP }>12 \mathrm{ng} / \mathrm{ml}+ \\
\text { PIVKA II }> \\
36 \mathrm{mAU} / \mathrm{ml}\end{array}$ \\
\hline Sensitivity & $60 \%$ & $78.6 \%$ & $92.5 \%$ \\
Specificity & $77.2 \%$ & $66.3 \%$ & $51.4 \%$ \\
PPV & $61.8 \%$ & $55.70 \%$ & $48.5 \%$ \\
NPV & $75.9 \%$ & $85.2 \%$ & $93.2 \%$ \\
\hline
\end{tabular}

PIVKA-II: Prothrombin induced by vitamin K absence-II; AFP: afetoprotein; HCC: hepatocellular carcinoma.

ultrasound. However, researches for the identification of an effective serum marker for screening of HCC are currently ongoing. AFP is widely used for this purpose, even though it is not recommended by neither EASL nor AASLD guidelines because of its low sensitivity and specificity (22). AFP is elevated in only $40-60 \%$ of cases of HCC. Sensitivity is even lower in early HCC cases, with serum elevation of AFP in only $10-20 \%$ of cases. Moreover, as a non-specific

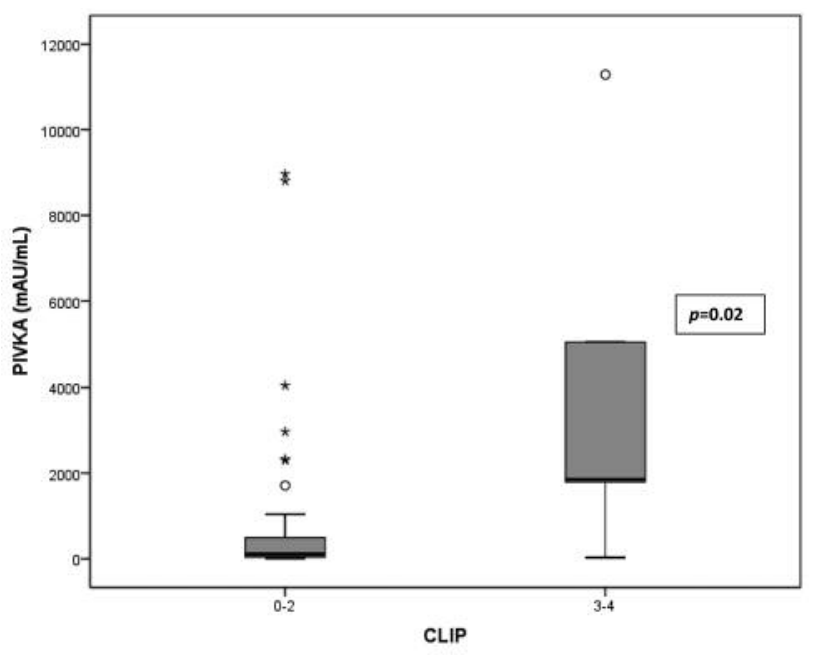

Figure 3. Box plot showing PIVKA-II levels according to CLIP stage. Patients were divided in two groups: the one who have a CLIP stage of 0-2 and the others who have a CLIP stage of 3-4. PIVKA-II: prothrombin induced by vitamin K absence-II: CLIP score (The Cancer of the Liver Italian Program score).

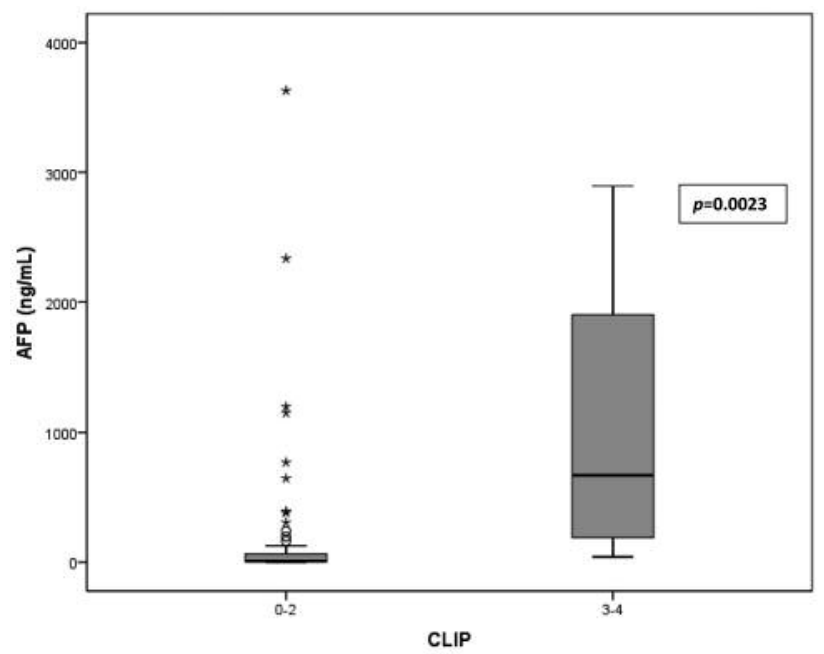

Figure 4. Box plot showing AFP levels according to CLIP stage. Patients were divided in two groups: the one who have a CLIP stage of 0-2 and the others who have a CLIP stage of 3-4. AAFP: $\alpha$-fetoprotein; CLIP score (The Cancer of the Liver Italian Program score).

inflammation and regeneration marker, it may also be elevated in liver diseases (e.g. liver cirrhosis and hepatic necrosis), as well as in some extra-hepatic conditions such as pneumonia and embryonal tumors (9). 
In our study, we showed that higher serum concentrations of both PIVKA-II and AFP, were significantly-related to diagnosis of HCC. This is in accordance with other authors, who showed the production of PIVKA-II by HCC and its role as an autologous growth, invasive and pro-angiogenetic factor (11-13) as well as its absence in non-neoplastic hepatic diseases (10).

Similarly to other authors (24), we did not observe any correlation between PIVKA-II and AFP serum levels. This allowed us to evaluate the combined performance of both markers in the diagnosis of HCC, as suggested by already available evidence $(19,24)$. When cut-off values of $36 \mathrm{mAU} / \mathrm{ml}$ for PIVKA-II and $12 \mathrm{ng} / \mathrm{ml}$ for AFP were selected, we showed that PIVKA-II, had a higher sensitivity but a lower specificity than AFP. Combining both markers further increased overall sensitivity and negative predictive value at the expense of specificity $(51.4 \%)$. These results slightly differ from a recent French study in which the authors showed that PIVKA-II had a better performance than AFP for early HCC diagnosis with a sensitivity of $77 \%$ (vs. 61\%), a specificity of $82 \%$ ( $v s .50 \%$ ), a positive predictive value (PPV) of $76 \%(v s .51 \%)$ and a negative predictive value (NPV) of $83 \%$ (vs. 62\%), at a cut-off values of $42 \mathrm{mAU} / \mathrm{ml}$ for PIVKA-II and $5.5 \mathrm{ng} / \mathrm{ml}$ for $\operatorname{AFP}(25)$.

In our cohort, both markers showed moderate diagnostic accuracy for HCC. Accuracy of PIVKA-II appeared to be slightly superior to AFP albeit without statistical significance. The serum concentrations of both markers increased at higher values of CLIP score.

In conclusion, PIVKA-II did not appear to be superior to AFP in screening for HCC in our cohort of patients. These markers appeared to perform best at cut-off values of $36 \mathrm{mAU} / \mathrm{ml}$ for PIVKA-II and $12 \mathrm{ng} / \mathrm{mL}$ for AFP. Nevertheless, the combination of PIVKA-II and AFP at the above mentioned cut-offs, may be considered as a screening test for HCC due to its high negative predictive value.

Further studies are needed to confirm our results in larger cohorts, especially in patients affected by liver cirrhosis of different etiologies.

\section{References}

1 WHO: GUIDELINES FOR THE SCREENING, CARE AND TREATMENT OF PERSONS WITH HEPATITIS C INFECTION, 2014.

2 Bruix J and Sherman M; American Association for the Study of Liver Diseases: Management of hepatocellular carcinoma: an update. Hepatology 53(3): 1020-1022, 2011.

3 El-Serag HB: Epidemiology of viral hepatitis and hepatocellular carcinoma. Gastroenterology 142(6): 1264-1273, 2012.

4 European Association For The Study Of The Liver and European Organisation For Research And Treatment Of Cancer: EASLEORTC Clinical Practice Guidelines: Management of hepatocellular carcinoma. J Hepatol 56(4): 908-943, 2012.
5 Ertle JM, Heider D, Wichert M, Keller B, Kueper R, Hilgard P, Gerken $G$ and Schlaak JF: A Combination of a-fetoprotein and des- $\gamma$-carboxy prothrombin is superior in detection of hepatocellular carcinoma. Digestion 87(2): 121-131, 2013.

6 Khattab M, Fouad M and Ahmed E: Role of biomarkers in the prediction and diagnosis of hepatocellular carcinoma. World $\mathbf{J}$ Hepatol 7(23): 2474-2481, 2015.

7 Farinati F, Marino D, De Giorgio M, Baldan A, Cantarini M, Cursaro C, Rapaccini G, Del Poggio P, Di Nolfo MA, Benvegnu L, Zoli M, Borzio F, Bernardi M and Trevisani F: Diagnostic and prognostic role of alpha-fetoprotein in hepatocellular carcinoma: both or neither? Am J Gastroenterol 101(3): 524-532, 2006.

8 Baek Y-H, Lee J-H, Jang J-S, Lee S-W, Han J-Y, Jeong J-S, Choi J-C, Kim H-Y and Han S-Y: Diagnostic role and correlation with staging systems of PIVKA-II compared with AFP. Hepatogastroenterology 56(91-92): 763-767, 2009.

9 Liebman HA, Furie BC, Tong MJ, Blanchard RA, Lo K-J, Lee S-D, Coleman MS and Furie B: Des- $\gamma$-carboxy (abnormal) prothrombin as a serum marker of primary hepatocellular carcinoma. N Engl J Med 310(22): 1427-1431, 1984.

10 Zhang Y-S, Chu J-H, Cui S-X, Song Z-Y and Qu X-J: Des- $\gamma-$ carboxy prothrombin (DCP) as a potential autologous growth factor for the development of hepatocellular carcinoma. Cell Physiol Biochem 34(3): 903-915, 2014.

11 Inagaki Y, Tang W, Makuuchi M, Hasegawa K, Sugawara Y and, Kokudo N: Clinical and molecular insights into the hepatocellular carcinoma tumour marker des- $\gamma$-carboxyprothrombin. Liver Int 31(1): 22-35, 2011.

12 Fujikawa T, Shiraha H and Yamamoto K: Significance of desgamma-carboxy prothrombin production in hepatocellular carcinoma. Acta Med Okayama 63(6): 299-304, 2009.

13 Koike Y, Shiratori Y, Sato S, Obi S, Teratani T, Imamura M, Yoshida H, Shiina S and Omata M: Des-gamma-carboxy prothrombin as a useful predisposing factor for the development of portal venous invasion in patients with hepatocellular carcinoma: a prospective analysis of 227 patients. Cancer 91(3): $561-569,2001$

14 Shirabe K, Itoh S, Yoshizumi T, Soejima Y, Taketomi A, Aishima $\mathrm{S}$ and Maehara $\mathrm{Y}$ : The predictors of microvascular invasion in candidates for liver transplantation with hepatocellular carcinoma - with special reference to the serum levels of des-gamma-carboxy prothrombin. J Surg Oncol 95(3): 235-240, 2007.

15 Hirokawa F, Hayashi M, Miyamoto Y, Asakuma M, Shimizu T, Komeda $\mathrm{K}$, Inoue $\mathrm{Y}$ and Uchiyama $\mathrm{K}$ : Outcomes and predictors of microvascular invasion of solitary hepatocellular carcinoma. Hepatol Res 44(8): 846-853, 2014.

16 Kim JM, Hyuck C, Kwon D, Joh J-W, Lee JH, Paik SW and Park CK: Protein induced by vitamin K antagonist-II (PIVKAII) is a reliable prognostic factor in small hepatocellular carcinoma. World J Surg 37(6): 1371-1378, 2013.

17 Poté N, Cauchy F, Albuquerque M, Voitot H, Belghiti J, Castera L, Puy H, Bedossa P and Paradis V: Performance of PIVKA-II for early hepatocellular carcinoma diagnosis and prediction of microvascular invasion. J Hepatol 62(4): 848-854, 2015.

18 Tanaka Y, Kashiwagi T, Tsutsumi H, Nagasawa M, Toyama T, Ozaki S, Naito $M$, Ishibashi $K$ and Azuma M: Sensitive measurement of serum abnormal prothrombin (PIVKA-II) as a marker of hepatocellular carcinoma. Hepatogastroenterology 46(28): 2464-2468, 1999. 
19 Kudo M: Clinical practice guidelines for hepatocellular carcinoma differ between Japan, United States, and Europe. Liver cancer 4(2): 85-95, 2015.

20 Zakhary NI, Khodeer SM, Shafik HE and Abdel Malak CA: Impact of PIVKA-II in diagnosis of hepatocellular carcinoma. J Adv Res 4(6): 539-546, 2013.

21 Okuda K: Epidemiology of primary liver cancer. In: Primary Liver Cancer in Japan. Springer (eds.), Tokyo, Japan, pp. 3-15, 1992.

22 Eslam M, Khattab MA and Harrison SA: Insulin resistance and hepatitis C: an evolving story. Gut 60(8): 1139-1151, 2011.

23 Cavani E, Rota CTT and Trenti T: Epatocarcinoma biomarcatori diagnostici e prognostici (Rassegna). Ligand Assay 19(2): 148$160,2014$.
24 Kuromatsu R, Tanaka M, Shimauchi Y, Shimada M, Tanikawa $\mathrm{K}$, Watanabe $\mathrm{K}$ and Yokoo T: Usefulness of ED036 kit for measuring serum PIVKA-II levels in small hepatocellular carcinoma. J Gastroenterol 32(4): 507-512, 1997.

Received May 5, 2017

Revised May 17, 2017

Accepted May 18, 2017 